\title{
Lumen
}

Selected Proceedings from the Canadian Society for Eighteenth-Century Studies

\section{La République des Lettres au féminin. Femmes et circulation des savoirs au XVIII ${ }^{\mathbf{e}}$ siècle}

\section{Marie-Laure Girou Swiderski}

Volume 28, 2009

URI : https://id.erudit.org/iderudit/1012035ar

DOI : https://doi.org/10.7202/1012035ar

Aller au sommaire du numéro

Éditeur(s)

Canadian Society for Eighteenth-Century Studies / Société canadienne d'étude du dix-huitième siècle

ISSN

1209-3696 (imprimé)

1927-8284 (numérique)

Découvrir la revue

Citer cet article

Girou Swiderski, M.-L. (2009). La République des Lettres au féminin. Femmes et circulation des savoirs au XVIII ${ }^{\mathrm{e}}$ siècle. Lumen, 28, 1-28.

https://doi.org/10.7202/1012035ar

Copyright (C Canadian Society for Eighteenth-Century Studies / Sociéte canadienne d'étude du dix-huitième siècle, 2009
Ce document est protégé par la loi sur le droit d'auteur. L'utilisation des services d'Érudit (y compris la reproduction) est assujettie à sa politique d'utilisation que vous pouvez consulter en ligne.

https://apropos.erudit.org/fr/usagers/politique-dutilisation/ 


\section{1 : La République des Lettres au féminin. Femmes et circulation des savoirs au XVIII ${ }^{\mathrm{e}}$ siècle}

Comment s'expliquer qu'au XXI siècle on puisse encore écrire sur le mouvement des Lumières comme si les femmes n'existaient pas, sans se préoccuper de la place qu'elles ont pu y tenir ou même en déclarant d'emblée qu'elles n'ont pu y en avoir aucune, sinon de sympathie ou d'opposition? En même temps, il est peu de termes plus en vogue actuellement que celui de "femme des Lumières» — et tout récemment même de «filles», hélas! Mais la plupart du temps, il ne semble pas du tout s'agir alors de marquer une appartenance, tout au plus une concomitance : elles se trouvent avoir vécu à cette époque-là, voilà tout. $\mathrm{Si}$ par contre, on veut désigner ainsi une femme qui, par son activité, sa façon de penser, s'inscrit dans le mouvement d'ouverture et de libération que s'est voulu le courant des Lumières, on comprend qu'André Magnan l'applique à Mme Bentinck ${ }^{1}$, ou qu'il désigne Mme du Châtelet dans une récente exposition de la $\mathrm{BNF}^{2}$. Tant d'autres noms de femmes méritant ce vocable nous viennent à l'esprit que leur absence en devient alors plus incompréhensible, quasi scandaleuse. Essayons donc de comprendre comment, malgré tant de recherches entreprises, tant d'études publiées depuis une trentaine d'années, on peut encore en être là.

1 André Magnan et Anne Soprani (textes présentés par), Une femme des Lumières, Écrits et lettres de la comtesse de Bentinck, CNRS Éditions, coll. « De l'Allemagne», 1997.

2 L'exposition s'intitule simplement «Mme du Châtelet» mais le terme «une femme des Lumières» suit son nom dans la présentation. 


\section{Un bel exemple d'invisibilité}

S'il est un cas où la présence et le rôle des femmes dans le mouvement des Lumières semblaient faire consensus, c'est bien celui des hôtesses des salons qui, d'un bout à l'autre du siècle, ont réuni des gens de divers horizons dans des rencontres que tous semblaient apprécier et qui scandaient l'actualité littéraire et intellectuelle du moment.

Or, on ne peut plus être sûr de rien, semble-t-il. Le livre d'Antoine Lilti, Le monde des salons ${ }^{3}$, vient de faire justice de cette croyance. Centrant son propos, comme le suggère le sous-titre, Sociabilité et mondanité à Paris au XVIII siècle, sur les comportements de la sociabilité mondaine, l'auteur s'ingénie à prouver que les salons n'ont pas été les laboratoires de pensée qu'on a longtemps cru; tout cela n'était qu'illusion et mythe ; la réalité fut tout autre, et les fameuses hôtesses n'eurent pas plus d'importance ou d'influence que n'importe quel grand ou bourgeois nanti se mêlant à la vie sociale du temps, qu'on nous montre très active. M. Lilti nous prouve, en effet, que ces réunions n'étaient pas l'apanage des dames, que des couples ou même des hommes seuls pouvaient aussi les animer.

Ce qu'on cherchait à établir jusqu'à présent dans les études sur ce sujet, c'est l'importance et la qualité de l'influence exercée par ces femmes sur le mouvement intellectuel et littéraire des Lumières. C'est à quoi s'employait déjà Benedetta Craveri dans son magnifique ouvrage, $L^{\prime}$ âge de la conversation ${ }^{4}$, très heureusement secondée en cela par la com-

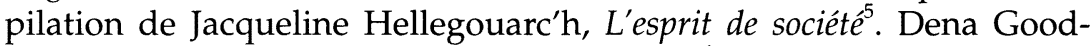
man, pour sa part, dans The Republic of Letters ${ }^{6}$, a même voulu faire des hôtesses des salons, les "salonnières " comme elle les nomme à tort, les chevilles ouvrières de l'expansion puis du triomphe des Lumières. Dans le livre de Lilti, au contraire, il s'agit de prendre l'exact contrepied de toute évaluation précédente. Ce parti pris d'opposition interdit que soit jamais envisagée l'éventuelle dimension intellectuelle de ce type de réunions, obstinément ramenées au contraire au rang d'échanges

3 Antoine Lilti, Le Monde des salons, Sociabilité et mondanité à Paris au XVIII siècle, Fayard, 2005.

4 Benedetta Craveri, L'Âge de la conversation, trad. de l'italien par É. Deschamps-Pria, Gallimard, 2001.

5 Jacqueline Hellegouarc'h, L'esprit de société. Cercles et «salons» parisiens au XVIII siècle, Garnier, 2000.

6 Dena Goodman, The Republic of Letters. A Cultural History of the French Enlightenment, Cornell University Press, 1994. 
mondains intéressés où la littérature et les idées jouent le rôle de simple divertissement. C'est bien pis encore dans un livre étroitement inspiré du sien. Jean de Viguerie dans son Filles des Lumières braque bien le projecteur sur les hôtesses, comme le suggère son sous-titre, Femmes et sociétés d'esprit à Paris; mais il le fait de telle façon qu'on en vient à se demander ce qui est pis de leur dissolution dans la pléthore, chez Lilti, ou de leur exaltation maladroite et paternaliste ici. Tout en soulignant, en effet, leur exceptionnelle qualité humaine, dont il semble moins convaincu par lui-même qu'obligé d'y croire par les témoignages des contemporains, Viguerie suggère qu'elles ne brillaient sans doute pas vraiment par leurs qualités intellectuelles. S'étant extasié sur leur exceptionnelle maîtrise de la langue française, il conclut en effet: "Cette parfaite connaissance leur permet sinon de comprendre toujours les propos tenus chez elles, tout au moins d'en deviner l'intérêt ${ }^{7}{ }^{\prime}$. Quant à leurs œuvres, il donne tout simplement au paragraphe qui en traite un titre qui en dit long: "Médiocrité assez générale de leurs écrits 》. Rappelons pour mémoire que se trouvent ainsi exécutées d'un même mouvement les admirables correspondances de Julie de Lespinasse et surtout de Mme Du Deffand, qui rivalisent presque en ampleur et en intérêt avec celle de Voltaire, les romans subversifs de Mme de Tencin, ceux de Mme Riccoboni et de Mme de Graffigny, ainsi que les œuvres morales de Mme de Lambert. On peut penser que cela fait tout de même beaucoup d'œuvres " médiocres"!

Mais A. Lilti ne les traite guère mieux. Dans son souci de vider ces relations de toute portée intellectuelle, il s'ingénie à dénier aux hô-

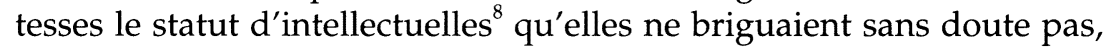
sauf à désigner par ce terme une femme qui aime penser, ce qu'elles étaient toutes. Prendre pour leur refuser cette qualité le seul exemple de Mme Geoffrin, notoirement inculte et se vantant de l'être, paraît un peu facile voire malhonnête. On sait bien en effet que Mme de Lambert et Mme Necker avaient toutes deux reçu au contraire une remarquable éducation. Quant à Mme Du Deffand ou Mme de Tencin, élevées au couvent, la seconde même ex-religieuse, elles possédaient l'instruction des dames de la bonne société. Mais cette prise de position permet de les ramener au seul rôle d'animatrices, de parfaites maîtresses de

7 Jean de Viguerie, Filles des Lumières, Femmes et sociétés d'esprit à Paris, D. Martin Morin, 2007, p. 75.

8 A. Lilti, « La femme du monde est-elle une intellectuelle? Les salons parisiens au XVIII ${ }^{\mathrm{e}}$ siècle » dans Nicole Racine et Gilbert Trebitsch (sous la direction de), Intellectuelles, Du genre en histoire des intellectuels, Éditions Complexe, 2004, p. 85-100. 
maison, de suggérer que finalement on vient chez elles surtout pour manger, car elles nourrissent abondamment tous ces hommes célèbres mais faméliques. Ces propos reviennent régulièrement dans les deux livres malgré leur fausseté évidente dans le cas de Julie de Lespinasse qui ne donne ni à manger ni à boire, faute de moyens, pendant les trois heures que durent les rencontres dans son salon. Si les intellectuels qui fréquentaient ces réunions ne cherchaient qu'à se sustenter et à se tailler une place dans la hiérarchie sociale, on comprend mal pourquoi nombre de familiers de Mme Du Deffand la désertent, elle et ses brillantes relations, elle et son rang social supérieur, pour aller dans l'entresol de Julie, célibataire, sans position sociale établie, désargentée et sans grande influence. N'est-ce pas parce qu'ils étaient sensibles malgré tout à son parfait usage du monde, à sa vive intelligence et à sa conversation stimulante?

Comment mesurer, en fin de compte, une influence quand elle passe essentiellement par la conversation : là est tout le problème. A. Lilti a sans doute raison de souligner que tous les témoignages dont nous disposons sur la question sont sujets à caution. S'ils émanent de membres des groupes concernés, on doit faire la part de la reconnaissance et de la nostalgie, comme de la rancœur quand le témoin fut un exclu. Et aucun document sonore, bien évidemment, ne viendra corroborer ou infirmer leurs propos. A. Lilti montre bien aussi comment, dès le début du XIX siècle avec Sainte-Beuve, et plus encore plus tard avec les Goncourt ${ }^{9}$, le monde fascinant des salons a fait l'objet d'une reconstruction nostalgique et éblouie, destinée à souligner par contraste le terne et l'étriqué de l'époque contemporaine.

Restent néanmoins certaines questions sans réponse, comme le pourquoi du passage de ces hôtesses à la postérité, elles et presque elles seules. Est-ce que cela compte qu'elles aient été toutes ( à l'exception de Mme Necker) des femmes seules, veuves ou séparées, sinon pour voir dans cette situation la raison d'être de leur activité mondaine, censée porter remède à l'ennui et à la solitude, comme l'avance J. de Viguerie ${ }^{10}$ ? Comment dans cette position de veuve ou de célibataire même, qui donne en effet plus de liberté mais rend en même temps plus fragile socialement, ont-elles pu acquérir un tel ascendant, un tel prestige ? Pourquoi Montesquieu choisit-il Mme de Tencin comme

9 Edmond et Jules de Goncourt, La femme au XVIII siècle, Firmin Didot, 1862.

10 Jean de Viguerie, Filles des lumières, op. cit. p. 71 : «ces reines des beaux-esprits sont en réalité des esseulées. Les sociétés d'esprit qu'elles forment et rassemblent chez elles, leur tiennent lieu des familles qu'elles n'ont pas.» 
une des toutes premières lectrices à qui faire parvenir $L^{\prime}$ Esprit des lois ? Pourquoi Diderot fait-il de Julie un personnage du Rêve de d'Alembert? Pourquoi enfin, sans rien publier, s'obstinent-elles à écrire, sinon parce $q u$ 'instruites et stimulées par ces échanges, elles aiment penser et apprendre? Comme un grand nombre de femmes à l'époque, en effet, les hôtesses ne publient rien. Certaines écrivent à peine, sauf des lettres, comme Mme Geoffrin; les autres, à l'instar de Mme de Tencin pour ses romans, publient anonymement. Seule Mme de Lambert publie, mais sur le tard et à contre-cœur.

Une des raisons qui peut expliquer la relative invisibilité des femmes dans l'histoire des Lumières, et partant une thèse comme celle d'A. Lilti, tient sans doute en grande partie aux modalités de leur participation active à la vie littéraire et intellectuelle.

\section{L'écriture invisible}

Nous avons peine à imaginer aujourd'hui l'impact du ridicule jeté, à la fin du XVII ${ }^{e}$ siècle, sur la femme savante par la pièce de Molière et tout un courant d'opposition au mouvement précieux. Mme de Lambert nous le rappelle au début de ses Réflexions nouvelles sur les femmes: «depuis ce temps-là, on a attaché presque autant de honte au savoir des femmes qu'aux vices qui leur sont le plus défendus ${ }^{11} . \gg$ Le coup a porté, c'est certain. Un leitmotiv revient, en effet, sous toutes les plumes féminines tout au long du siècle, reprenant la leçon de Fénelon. C'est encore Mme de Lambert qui remarque dans ses Avis à sa fille : «songez que les filles doivent avoir sur les sciences une pudeur presque aussi tendre que sur les vices ${ }^{12}$ ». Ce n'est donc pas tant écrire qui leur est interdit que montrer qu'elles savent, en écrivant puis en publiant.

Quand nous apprenons aujourd'hui la réaction violente de Mme de Lambert découvrant que ses amis ont donné ses textes à un éditeur, quand nous lisons le refus indigné de Mme Du Deffand de voir son nom paraître dans un texte de Walpole, nous sommes tentés d'interpréter ces comportements comme de la pose, d'y voir une coquetterie d'auteur. Ce serait une erreur mais d'abord, surtout, un anachronisme. Même si les choses avaient bien changé dans le monde de l'édition à la fin du siècle, on voit encore Mme Roland persister dans le refus de publier et, au début du XIX ${ }^{e}$ siècle encore, Mme de Staël pourra tracer dans

11 Mme de Lambert, "Réflexions nouvelles sur les femmes ", dans CEuvres de Madame la Marquise de Lambert, rassemblées pour la première fois, Amsterdam, 1747, p. 174. 
De l'influence des passions et De la littérature ${ }^{13}$, entre autres, le tableau épouvantable des malheurs qui attendent toute femme qui ose risquer la publication.

Une confirmation de cette hantise du ridicule sinon du déshonneur peut être tirée de la fréquence des publications féminines sous anonymat. Et pourtant cette tactique est fort peu efficace. Dans cette société oisive, ce petit monde finalement, où l'on n'aime rien tant que se dérober aux autres tout en réussissant à surprendre leur secret, le voile tombera vite. Voici comment Mme Thiroux d'Arconville résume ce qui se passe alors : "affichent-elles la Science ou le bel esprit ? Si leurs ouvrages sont mauvais, on les siffle, s'ils sont bons, on les leur ôte, il ne leur reste que le ridicule de s'en être dit les auteurs ${ }^{14} . »$ Mme Roland ne dira pas autre chose. Et elle a raison, comme le montrent, encore de nos jours, les innombrables tentatives de découverte du véritable auteur de La Princesse de Clèves, qui pour beaucoup ne peut toujours pas être Mme de La Fayette.

L'autre obstacle qui limite le libre accès des femmes à l'écriture et à la publication, c'est la faiblesse de l'éducation féminine. Et non seulement sa faiblesse mais surtout son étroitesse, la hantise des éducateurs de voir corrompre l'innocence féminine par l'instruction ${ }^{15}$. On redoute même les mathématiques qui pourraient les gâter en les rendant orgueilleuses si par malheur elles y comprenaient quelque chose ! Pendant longtemps, elles n'apprennent à lire et à écouter même que l'Histoire sainte ou les ouvrages édifiants. Si, d'ailleurs, on voulait élargir leur horizon en variant leurs lectures, les éducateurs seraient bien embarrassés et devraient se rabattre sur les ouvrages à l'usage des garçons (Mme de Maintenon avait bien vu cette carence à Saint-Cyr, c'est pourquoi elle $\mathrm{s}^{\prime}$ était attelée à la composition de textes à $\mathrm{l}^{\prime}$ intention des jeunes filles ${ }^{16}$.) Dans les couvents, pendant longtemps, on limite au minimum le savoir

13 Mme de Staël, De l'influence des passions, " De la vanité »; De la littérature, chapitre IV, « Des femmes qui cultivent les lettres ».

14 Marie Geneviève Charlotte Thiroux d'Arconville, Mélanges de littérature, de morale et de physique, Amsterdam, 1775, "Sur les femmes ", p. 370-371.

15 Sur toutes ces questions, il y a bien sûr la somme de Linda Timmermans, L'Accès des femmes à la culture sous l'Ancien régime, Paris, Honoré Champion, coll. "Champion Classiques ", 2005, mais aussi le livre de Martine Sonnet, L'Éducation des filles au temps des Lumières, Paris, Cerf, 1987, et celui de Paule Constant, Un monde à l'usage des demoiselles, Paris, Gallimard, 1987.

16 Jacques Prévot, La Première institutrice de France, Madame de Maintenon, Paris, Belin, 1981; voir entre autres p. 219-250. 
communiqué $^{17}$. Pourtant il y a deux talents qu'on leur enseigne de mieux en mieux : à écrire des lettres et à mieux comprendre les textes qu'on leur lit en rédigeant des extraits. Ces deux exercices serviront d'outils à toutes celles (et elles sont nombreuses, en effet) qui s'efforcent de compléter, seules ou en cherchant de l'aide, le savoir que ne leur a pas procuré leur éducation. La carence du système se vérifie indirectement à cette mention qu'on trouve fréquemment quand on étudie la vie des femmes à l'époque : «elle avait reçu une excellente éducation» - ce qui montre que ce n'était pas encore la norme. Mme d'Épinay avoue qu'elle ne savait pas grand chose quand elle s'est mariée, ce qui ne l'empêchera pas ensuite d'écrire des textes remarquables et de tenir honorablement sa partie dans l'équipe de la Correspondance littéraire de Grimm et Meister. Quant à Mme Geoffrin, la plus célèbre des hôtesses, son manque d'instruction est notoire. Elle est la première à le dire et vante l'exemple de sa grand-mère, femme très intelligente qui n'avait guère fréquenté l'école. Cet exemple rappelle la nécessité de distinguer ignorance et sottise. Les critiques ont vite fait de conclure, malgré de nombreux exemples du contraire, que les femmes du XVIII ${ }^{e}$ siècle ne pouvaient être très intelligentes du fait de leur instruction indigente.

Sans doute faut-il rappeler la position de Poullain de la Barre qui souligne l'aptitude supérieure des femmes à accéder à la vérité parce que leur jugement n'a pas été gâté, ni leur esprit encombré d'une masse de faux savoir abrutissant. Et puis les femmes sont curieuses. Or «la curiosité est une connaissance commencée qui vous fait aller plus loin et plus vite dans le chemin de la vérité ${ }^{18}$ ", souligne-t-il. Mme de Lambert reprend cette pensée ${ }^{19}$ que Marivaux n'oubliera pas : dans La Vie de Marianne, l'hérö̈ne affirme qu' «il n'y a que le sentiment qui nous puisse donner des nouvelles un peu sûres de nous ${ }^{20}{ }^{\prime}$.

Les femmes redoutent donc la publication mais elles ne cessent pas pour autant d'écrire. D'abord des lettres, surtout des lettres familières, comme on les nomme, et qu'elles plieront à toutes sortes d'usages. La lettre, substitut de la conversation, tenant lieu de la visite qu'on ne peut pas rendre, sert comme cette dernière à entretenir le tissu social

17 A ce sujet, voir Martine Sonnet, op. cit., et Dominique Godineau, Les Femmes dans la société française $16^{e}-18^{e}$ siècle, Paris, Armand Colin, coll. "U. Histoire », 2003, spécialement au chapitre 6, p. 124.

18 François Poullain de la Barre, De l'égalité des sexes, Paris, Dupuis, 1673.

19 Dans Avis d'une mère à sa fille, p. 80, elle ajoute : «c'est un penchant de la nature qui va au-devant de l'instruction; il ne faut pas l'arrêter par l'oisiveté et la mollesse.»

20 Marivaux, La Vie de Marianne, Garnier, p. 22. 
sans lequel on ne peut pas survivre à l'époque, surtout avec un nom à illustrer et un rang à conserver. Elle sert aussi à communiquer des connaissances de toutes sortes. Elle se fait volontiers chronique, donnant des nouvelles du monde pour ceux qui n'ont pas la chance d'être à Paris ou près des cercles du pouvoir. Et cela donne, par exemple, le surprenant échange, souvent politique, de deux femmes en pleine crise parlementaire : la correspondance de Mme Durey de Meinières avec la marquise de Lénoncourt ${ }^{21}$. On peut penser aussi aux innombrables correspondantes de Voltaire qui lui permettent de prendre le pouls de la capitale et de la cour, comme Mme Du Deffand ou la marquise de la Tour du Pin.

Les épistolières assignent aux lettres les rôles les plus divers. Il suffit de voir les différences de ton et d'écriture d'une même scriptrice. Estce bien la même marquise Du Deffand qui écrit à Mme de Choiseul, à Voltaire ou à Walpole, en contrôlant mal sa passion ? Les lettres deviennent une école de savoir-vivre pour les plus jeunes, comme le montrent les lettres de Julie de Lespinasse à Condorcet ${ }^{22}$. Elles peuvent devenir un véritable laboratoire où s'affirme une pensée scientifique : voir les lettres de Mme Du Châtelet à Maupertuis, Jean Bernouilli ou Clairaut ${ }^{23}$. Entre une femme mûre et un homme plus jeune, l'échange se colore d'une touche maternelle dont la passion n'est pas toujours absente : on le voit bien dans les lettres de Mme de Charrière à Benjamin Constant ${ }^{24}$, ou celles de Mme Riccoboni à Robert Liston ${ }^{25}$. L'échange est intellec-

21 Marie-Laure Girou Swiderski, «De la 'gazette' au 'commerce des âmes', les lettres de la présidente de Meinières à la marquise de Lénoncourt», dans Marie-France Silver et Marie-Laure Girou Swiderski (textes réunis par), Femmes en toutes lettres, Les épistolières $d u$ XVIII siècle, Oxford, Voltaire Foundation, coll. "Studies on Voltaire and the Eighteenth Century / SVEC », 2000, p. 119-139, et "Lettres de Mme de Meinières à Mme de Lénoncourt (1770-1774) ", dans Lettres de femmes, Textes inédits et oubliés du XVI' au XVIII siècle, Paris, Honoré Champion, coll. "Textes de la Renaissance. Série 'Éducation des femmes' ", 2005, p. 379-420.

22 Julie de Lespinasse, Lettres à Condorcet, éd. présentée et annotée par J.-N. Pascal, Paris, Desjonquères, coll. «XVIIIe siècle », 1990.

23 Roland Bonnel, «La correspondance scientifique de la marquise Du Châtelet : la 'lettre laboratoire'», dans Femmes en toutes lettres, Les épistolières du XVIII siècle, Oxford, Voltaire Foundation, coll. «Studies on Voltaire and the Eighteenth Century / SVEC », 2000, p. 79-95.

24 Isabelle de Charrière, Benjamin Constant, Correspondance (1787-1805), édition établie, préfacée et annotée par J.-D. Candaux, Paris, Desjonquères, coll. « XVIIIe siècle », 1996. 
tuel et intime à la fois entre Mme d'Épinay et l'abbé Galiani ${ }^{26}$, ou plus encore entre Voltaire et Mme Bentinck: trois cent lettres de lui, une trentaine seulement d'elle ( il ne les a pas gardées!) mais qui suffisent à nous dévoiler son intelligence, sa connaissance du monde, sa remarquable qualité humaine ${ }^{27}$. On y découvre aussi une curieuse dimension quasi éducative ; pleine de franchise et de délicatesse, elle ose parfois lui prodiguer ses conseils, comme dans cette lettre de mars 1753, au moment où il s'apprête à fuir Frédéric après le scandale d'Akakia:

Au nom de Dieu et de la fermeté que vous m'avez toujours vue à vous conserver mon estime et ma reconnaissance, lisez de sang-froid ce que je vous écris aujourd'hui ; le pas que vous allez faire à présent à Postdam est décisif et sans remède. Ne vous trompez pas, vous n'êtes pas assez fort pour renoncer pour toujours à lui ; je vous ai mieux lu que vous-même, et c'est une illusion d'une passion irritée qui vous aveugle, si elle vous persuade que vous avez [assez] de dépit pour vous guérir de l'ascendant qu'il a pris sur votre cœur. [...] Cédez de bonne grâce au sentiment joint à la raison et à tous vos intérêts réunis. Il est cruel de perdre la bienveillance d'un souverain et la considération qui la suit, mais il est affreux de perdre un ami. Grandeur, gloire et plaisir, tout se réunissait pour vous auprès de lui. Voilà comme le monde entier raisonne avec moi, voilà comme vous raisonnerez vous-même un jour, quand un jour vous serez de sang-froid : puissiez-vous l'être assez tôt pour ne vous point préparer d'éternels regrets $^{28}$.

Outre les lettres, elles sont de plus en plus nombreuses tout au long du siècle à céder à la tentation d'écrire leur vie, leurs aventures, et bien sûr la Révolution leur donnera toutes les raisons de vouloir le faire. Dans ces Mémoires, certaines vont évoquer les figures historiques qu'elles ont côtoyées, comme Mme de Pompadour (Mme du Hausset) ${ }^{29}$

1783), éd. James C.Nicholls, Oxford, Voltaire Foundation, coll. «Studies on Voltaire and the Eighteenth Century », 1976.

26 Ferdinando Galiani, Louise d'Épinay, Correspondance, texte établi et annoté par D. Maggetti, en collaboration avec G. Dulac, 4 vol., Desjonquères, coll. "XVIIIe siècle ", 1992-1995.

27 Voltaire et sa "grande amie " Correspondance complète de Voltaire et Mme Bentinck (1740-1778), édition de F. Deloffre et J. Cormier, Oxford, Voltaire Foundation, coll. « Vif », 2003.

Ibid., p. 187.

29 Mémoires de Madame du Hausset sur Louis XV et Mme de Pompadour, Paris, Mercure de France, coll. « Le Temps retrouvé », 1985. 
ou Marie-Antoinette ( Mme Campan) ${ }^{30}$; d'autres retracent simplement une vie fertile en rebondissements de tous ordres, comme Mme de la Tour du Pin ou Mme de la Rochejaquelein. ${ }^{31}$ A l'exception de Mme de Genlis qui, les ayant écrits pour se justifier, en commence la publication avant même de les avoir achevés, aucune ne songe d'abord à publier.

Beaucoup en effet écrivent pour elles-mêmes, par besoin ou pour le plaisir. On pense à Mme Necker ${ }^{32}$, interdite de publication sinon même d'écriture par son mari et qui passera pourtant toute sa vie à noircir des pages où elle note méticuleusement ses moindres pensées. Il est vrai que, très croyante et compulsivement perfectionniste, elle se donne alors l'alibi de la connaissance de soi nécessaire pour travailler à son perfectionnement moral. Ses œuvres seront partiellement publiées, à titre posthume, par son mari sous le titre Mélanges et Nouveaux Mélanges $^{33}$. Bien différent est le cas de Mme Thiroux d'Arconville, polygraphe, publiant tout au long de sa vie les textes les plus divers, traductions, œuvres morales, ouvrages scientifiques et historiques et même, hélas!, quelques romans. Après la Révolution, elle ne publie plus rien mais continue pourtant d'écrire jusqu'à sa mort en 1805, à quatre-vingts $a^{34}{ }^{34}$. Durant ces années, elle rédige douze volumes de Pensées, réflexions et anecdotes de Mme d'Arconville qu'elle fera relier sans les publier. On les a crus longtemps perdus. Ils viennent de refaire surface à l'île Maurice et nous espérons pouvoir bientôt en exploiter la riche diversité, grâce à un CD-Rom.

30 Mémoires de Mme Campan, première femme de chambre de Marie-Antoinette, Paris, Mercure de France, coll. « Le Temps retrouvé », 1988.

31 Mémoires de la marquise de la Tour du Pin, Journal d'une femme de cinquante ans, 17781815, Paris, Mercure de France, coll. "Le Temps retrouvé », 1989; Mémoires de la marquise de la Rochejaquelein (1772-1857), Paris, Mercure de France, coll. « Le Temps retrouvé », 1984.

32 Dena Goodman, "Suzanne Necker's Melanges: Gender, Writing and Publicity » dans Elizabeth C. Goldsmith and Dena Goodman (ed.), Going Public: Women and Publishing in Early Modern France, Cornell University Press, coll. «Reading Women Writing», 1995, p. 210-223.

33 Mélanges extraits des manuscrits de Mme Necker, Pougens, Paris, 1798.

34 Marie-Laure Girou Swiderski, «Vivre la Révolution. L'incidence de la Révolution sur la carrière et la vie de trois femmes de lettres», dans Marie-France Brive (édition préparée par), Les Femmes et la Révolution Française, Presses universitaires du Mirail, Toulouse, 1990, vol. 2, p. 239-249; et «Écrire à tout prix, Mme Thiroux d'Arconville, polygraphe», Inventaire raisonné de la prose féminine non-fictionnelle, http://aix1.uottawa.ca/-margirou.html. 


\section{Et pourtant elles publient...}

Pour celles que la publication inquiète et qui ne se fient pas à l'anonymat, il reste une solution : traduire.

Il semble bien clair, en effet, que pour la période de l'Ancien régime (et ce sera encore vrai au XIX ${ }^{\mathrm{e}}$ siècle) la traduction fut la voie royale et sûre d'accès des femmes à l'écriture. Elle offre un double (sinon un triple) avantage. Cette activité est dans le droit fil du rôle traditionnel des femmes, êtres pour autrui : elles se mettent au service de la pensée d'un autre et en outre, elles sont utiles à leurs semblables, leur rendant disponibles des connaissances auxquelles ils n'auraient pas pu avoir accès. En même temps, elles préservent leur anonymat. Surtout, cet exercice leur permet de s'intruire, même dans des domaines habituellement interdits aux femmes: si la plupart d'entre elles traduisent des romans, comme Mme Riccoboni, et des textes littéraires ou moraux, comme Mme Belot et Mme Thiroux d'Arconville, certaines se risqueront de plus en plus, à mesure que le siècle avance, à traduire des ouvrages de philosophie, d'histoire, de science et même de médecine. La traduction leur offre aussi parfois l'occasion, en participant à l'établissement de l'apparat critique, de donner discrètement des preuves de leur savoir. Ainsi nombre des traductrices scientifiques dont nous parlerons plus tard sont suffisamment expertes dans ce domaine pour compléter le texte et même en corriger les erreurs, comme Mme Thiroux d'Arconville.

Presque toutes traduisent surtout de l'anglais. Rappelons que c'est l'époque des «belles infidèles » : le respect scrupuleux du texte n'est pas la norme. La critique a tendance à s'acharner sur celles qui osent s'attaquer à de véritables monuments, comme Mme Belot qui a entrepris de traduire l'Histoire d'Angleterre de Hume. La Correspondance littéraire a beau jeu à rappeler quelques-uns des gros contre-sens qu'elle a pu commettre ${ }^{35}$. Hume semble pourtant avoir été assez satisfait de sa traductrice pour la recommander à ses amis et lui demander de traduire les Stuart, la dernière partie de l'Histoire, bien négligemment traduite auparavant par l'abbé Prévost.

Anonymes, fortes de l'utilité de leur tâche, ces traductrices osent pourtant timidement donner en marge quelques pages de leur cru, au moins pour expliquer les principes qui ont présidé à leur traduction : ainsi Mme Thiroux d'Arconville ne craint pas d'avouer, dans la préface

35 Grimm, Diderot, Raynal, Meister, Correspondance littéraire, philosophique et critique, Garnier, 1878. 
de sa traduction des Mélanges de littérature anglaise, les libertés qu'elle a osé prendre avec les textes traduits, décidément trop désordonnés, à son goût, pour l'esprit français ! Mme Belot ira plus loin : elle fait précéder sa traduction du Rasselas de Samuel Johnson d'une véritable dissertation sur le bonheur ${ }^{36}$.

Pour s'inscrire dans l'histoire littéraire et intellectuelle de leur temps, les femmes ont su prendre le système à son propre piège. Puisque la société ne leur reconnaît vraiment qu'un seul rôle légitime, celui de mère, et qu'une seule forme de compétence " naturelle ", celle qu'exige l'éducation des enfants, elles s'empressent de publier sur ce sujet. Aussi trouve-t-on une grande variété de contributrices sur le thème, alors brûlant, de l'éducation féminine - et tout d'abord des pédagogues de métier, en quelque sorte, comme Mme Leprince de Beaumont ou Mme de Genlis et, à la fin du siècle, Mme de Miremont ou Mme Campan, devenue sous Napoléon directrice d'une maison d'éducation; toutes tablent sur la compétence acquise dans l'exercice de leurs fonctions de gouvernante. Les deux premières surtout publient massivement : Mme Leprince de Beaumont ses divers Magasins des enfants, des adolescentes ${ }^{37}$, etc, qui connurent maintes rééditions au XIX ${ }^{\mathrm{e}}$ siècle; Mme de Genlis, un roman pédagogique, Adèle et Théodore, mais aussi des ouvrages de morale pour enfants, Les Veillées $d u$ château ${ }^{38}$, et des pièces de théâtre enfantines. A leur tour, des grandes dames vont s'essayer dans cette veine. Au début du siècle, Mme de Lambert publie ses Avis d'une mère à son fils, puis à sa fille; Mme d'Épinay, elle, s'inspire de l'expérience acquise en éduquant sa petite fille pour écrire ses remarquables Conversations $d^{\prime}$ Émilie $^{39}$. Toutes sont sûres, ce faisant, de se rendre utiles; elles savent en effet d'expérience à quel point l'éducation féminine est négligée et quelle pénurie doivent affronter celles qui voudraient se charger

36 Samuel Johnson, Histoire de Rasselas, prince d'Abyssinie, Desjonquères, 1994, coll. "XVIII siècle »,p. 17-22. Voir dans le même volume l'article d'Annie Rivara, "Notes sur les traductions de Rasselas par Mme Belot et par le comte de Fouchecour », p. 176-185.

37 Mme Leprince de Beaumont, Le Magasin des enfants ou dialogues d'une sage gouvernante avec ses élèves, Senlis, 1828; Magasin des jeunes dames qui entrent dans le Monde, se marient, leurs Devoirs dans cet Etat $\mathcal{E}$ envers leurs Enfants : Pour faire suite au Magasin des Adolescentes, Londres, 1764.

38 Mme de Genlis, Les Veillées du château ou cours de morale à l'usage des enfants, Maradan, 1816.

39 Louise d'Épinay, Les Conversations d'Émilie, texte présenté par R. Davison, Oxford, Voltaire Foundation, coll. "Studies on Voltaire and the Eighteenth Century", 1996. 
elles-mêmes de l'éducation de leurs enfants, comme Rousseau a semblé les y inciter.

Il est un autre domaine où les femmes se sentent une certaine compétence et croient pouvoir jouer un rôle utile, c'est la morale. Elles vont donc se risquer à partager leur expérience, à communiquer le savoir durement acquis sur ce que cela veut dire d'être femme dans ce monde et en ce temps. Mme de Lambert écrit ainsi ses Réflexions nouvelles sur les femmes, un Traité de la vieillesse, un autre de l'Amitié. Mme de Puisieux écrit des Caractères ( souvent attribués à Diderot) et Mme Du Châtelet rédige un délicieux Discours sur le bonheur ${ }^{40}$, publié après sa mort.

Parmi les autres textes publiés en leur temps, souvent œuvre unique d'une auteure aujourd'hui totalement inconnue, on trouve une grande variété d'œuvres ${ }^{41}$ dont certaines prises de position sur l'éternelle question des femmes: en 1750, la mystérieuse Mlle Archambault ${ }^{42}$ défendait la supériorité des femmes, comme à l'aube de la Révolution, Mme de Cambis ${ }^{43}$ ou Mme Gacon-Dufour ${ }^{44}$ prôneront l'égalité des sexes; cette dernière essayera aussi de contrer le projet de Sylvain Mareschal qui, en 1800, propose d'interdire aux femmes d'apprendre à lire! Parmi de nombreuses œuvres de circonstance, se signalent les textes proposés aux concours des Académies ; c'est ainsi qu'à la veille de la Révolution, Mme de Charrière ${ }^{45}$, Mme Roland et Mme de Staël soumettront toutes trois des études sur Jean-Jacques Rousseau. Mme Belot, pour sa part, publie en 1756 ses Réflexions d'une provinciale sur le discours de J.J. Rousseau touchant l'inégalité des conditions et en 1758, des Observations sur la noblesse et le tiers état où elle prend parti dans la querelle de la noblesse commer-

40 Madame du Châtelet, Discours sur le bonheur, Paris, Payot et Rivages, coll. « Rivages poche/Petite bibliothèque ", 1997.

41 Marie-Laure Girou Swiderski, «Surprises et leçons d'un inventaire : la prose féminine non-fictionnelle au $18^{\text {e }}$ siècle», Dix-huitième siècle, Femmes des Lumières $n^{\circ} 36$, 2004, p. 171-187.

42 Mlle Archambault, Dissertation sur la question: Lequel de l'homme ou de la femme est plus capable de constance? ou la cause des dames soutenue par Mlle A. contre $M^{* * *}$ et $M$. L. L. R., Paris, Vve Pissot et J. Bullot, 1750.

43 Mme de Cambis, Du sort actuel des femmes (par Mme de Cambis), Impr. Du Cercle social, 1791.

44 Marie-Armande Gacon-Dufour, Mémoire pour le sexe féminin contre le sexe masculin (1787), dans Opinions de femmes de la veille au lendemain de la Révolution française, préface de G. Fraisse, Paris, Côté-Femmes, 1989.

45 Mme de Charrière, Éloge de J.-J. Rousseau, qui a concouru pour le prix de l'Académie française, Grégoire, 1790. 
çante, après l'abbé Coyer et le chevalier d'Arcq. Ces divers exemples suggèrent, comme le confirme l'étude de Carla Hesse, que la publication est devenue plus accessible pour les femmes au cours du siècle ${ }^{46}$.

\section{Les romancières}

Pour celles qui veulent ou doivent écrire parce qu'elles espèrent en vivre, le roman semble le choix le meilleur - parce que depuis un siècle des femmes s'y sont illustrées mais aussi, hélas!, parce qu'il reste un genre considéré comme bas et facile, donc destiné et accessible aux femmes. Mais en devenant romancières, elles savent qu'elles s'exposent à toute la virulence de la critique, surtout que, bien souvent, leur position sociale de roturière, veuve ou séparée, les rend encore plus vulnérables. La Correspondance littéraire, en particulier, ne cesse de tomber à bras raccourcis sur toutes les malheureuses qui osent s'essayer au roman. La Bibliographie du genre romanesque de Frautschi, Martin et Mylne, bien que débutant en 1751, recense cent noms de femmes, dont près de la moitié ont publié une seule œuvre. Elles s'y sont essayées souvent faute d'autres moyens honnêtes de survivre. Elles n'avaient plus rien à perdre et publier apparaît alors simplement comme une transgression de plus les désignant au mépris public.

Pour quelques exceptions, ce peut être au contraire une seconde chance. Ainsi en va-t-il pour Mme Riccoboni, qui se lance dans la carrière à la cinquantaine, après avoir été actrice vingt ans sans grand succès. Elle sait ce que c'est qu'être exposée au regard public, et de toutes façons il faut vivre. Or ses œuvres vont connaître un grand succès et lui valoir respect et notoriété de bon aloi, même de la part des critiques. L'échange épistolaire qui l'oppose à Choderlos de Laclos à l'occasion des Liaisons dangereuses ${ }^{47}$, même s'il est teinté de la part du romancier d'une certaine ironie condescendante, en dit long sur la célébrité qu'elle a su gagner. Dès ses premiers romans, la critique la traite avec égards. Voici ce qu'écrit Raynal dans la Correspondance littéraire à propos de l'Histoire de Miss Jenny en juin 1764 :

46 Carla Hesse, «French women in print, 1750-1800 : an essay in historical bibliography» dans Haydn T. Mason (éd.), The Darnton Debate. Books and Revolution in the Eighteenth Century, Oxford, Voltaire Foundation, coll. «Studies on Voltaire and the Eighteenth Century ", 1998, p. 65-82.

47 Choderlos de Laclos, "Correspondance de Laclos et de Madame Riccoboni au sujet des Liaisons dangereuses», dans CEuvres complètes, éd. L. Versini, Paris, Gallimard, coll. « Bibliothèque de la Pléiade », 1967, p. 686-698. 
L'art de narrer avec beaucoup de concision et de rapidité, celui de semer dans son récit des réflexions fines et justes, beaucoup de finesse et de grâce dans le style et un ton très distingué, voilà les principales qualités de la plume de Mme Riccoboni.

Ce qui ne l'empêche pas de voir en Miss Jenny un roman invraisemblable et médiocre.

Un ton résolument féministe (voir la fameuse dernière lettre ouverte de Fanny dans Lettres de Fanny Butlerd), un constant souci de renouveler l'image du personnage féminin, une peinture touchante et vraie des aléas de la condition féminine, surtout celle des femmes mariées (Lettres de milady Juliette Catesby), un ton personnel qui vise à impliquer les lectrices dans la diégèse : voilà autant d'atouts qui ont permis à Mme Riccoboni de renouveler le roman sentimental et de lui donner ses marques de noblesse. On peut se demander si Rousseau aurait choisi la forme épistolaire pour La Nouvelle Héloïse si Mme Riccoboni n'avait pas eu autant de succès avant lui.

Celles que ne tentent ni les traités de morale ni les œuvres autobiographiques s'efforcent de faire passer dans le roman, en le transformant au besoin, leur vision du monde et le poids de leur expérience personnelle. A la différence de Mme de Genlis ou de Mme Leprince de Beaumont, Mme de Charrière n'a jamais écrit de traité pédagogique, bien qu'elle assume remarquablement dans sa correspondance un rôle de mentor auprès de ses jeunes amies. Mais tout au long de son œuvre romanesque, elle poursuit discrètement sa réflexion sur les conditions d'une bonne éducation féminine : dans les Lettres écrites de Lausanne, par exemple, elle évoque la dyade formée par Cécile et sa mère qui repousse vigoureusement le modèle d'éducation encyclopédique d'Adèle et Théodore de Mme de Genlis. On peut au contraire percevoir dans cette relation mère-fille comme un écho fraternel de la démarche pédagogique de Mme d'Épinay dans les Conversations d'Émiliee ${ }^{48}$, refusant la figure d'une éducatrice infaillible et toute puissante, selon le modèle de l'Émile, au profit d'un dialogue vrai avec l'enfant, où l'adulte reçoit autant qu'il donne. Dans Sainte-Anne, s'interrogeant sur les bienfaits discutables de la culture, elle poursuit sa réflexion sur les rapports obligés entre éducation morale et instruction et peint une héroïne analphabète mais pleine de sens et d'un savoir autre. Enfin, dans Trois femmes, elle s'oppose à

48 Mélinda Caron, «L'intimité pédagogique dans les Conversations d'Émilie de Louise d'Épinay», dans M-L. Girou Swiderski (dir). Terrae incognitae de l'écriture féminine, Ottawa/Toronto/New York, Éditions Legas, 2004, p. 97-105. 
l'impératif kantien en représentant des femmes imparfaites mais remplies de remarquables qualités humaines.

Voulant, elle aussi, édifier, Mme Élie de Beaumont, dans ses Lettres $d u$ marquis de Roselle, ose peindre une jeune fille lucide et quasi parfaite réussissant finalement à arracher le héros naïf aux manœuvres d'une fille. Bien que son roman connaisse un grand succès, ses efforts ne lui vaudront que ce jugement de la Correspondance littéraire :

On ne peut refuser son estime à celle qui a écrit les Lettres du marquis de Roselle mais on l'estimerait davantage, si les ayant lues elle les avait jetées au feu en les jugeant indignes de la publication.

Le pragmatisme et la volonté d'ancrer la fiction dans une expérience réelle paraissent les qualités principales des meilleurs romans écrits par des femmes. De la dénonciation iconoclaste des horreurs de la condition féminine dans Mémoires du comte de Comminge de Mme de Tencin à l'odyssée exemplaire de Zilia chez Mme de Graffigny, on retrouve le même écho de l'expérience féminine. Les critiques se sont gaussés du parcours pénible de la péruvienne ${ }^{49}$, privée de moyens de communication et devant refaire dans une langue étrangère l'apprentissage de l'enfant, rappel pathétique de l'éveil de la statue de Condillac. Les Persans de Montesquieu, eux, n'avaient aucun problème de communication pendant leur séjour en France où ìls soutenaient sans peine en français la conversation sur les sujets les plus ardus. On peut voir sans doute, dans cet aspect des Lettres d'une Péruvienne, une transposition de la dure expérience personnelle de la romancière, Lorraine peinant à se faire admettre dans les milieux mondains de la capitale. Mais ce périple quasi initiatique est aussi une belle parabole des affres de la conquête de l'identité féminine, pour celles qui se sentent souvent étrangères dans le monde des hommes.

Dans un tout autre registre, qui peut lui avoir été inspiré par les défaillances morales de Louis XV, Mme Leprince de Beaumont poursuit sur le mode utopique, dans Civan, roi de Bongo, sa réflexion sur l'éducation idéale d'un prince. Si son roman évoque parfois le Télémaque de Fénelon, on remarque qu'elle a l'audace d'imaginer que le pédagogue idéal serait une femme, à laquelle, il est vrai, elle a la prudence de donner des attributs quasi divins sans doute destinés aussi

49 Henri Coulet la juge "parfois niaise» dans Le Roman jusqu'à la Révolution, t. 1, Armand Colin, coll. «U », 1967, p. 383. 
à justifier le pouvoir parfois presque sadique qu'elle exerce arbitrairement sur son royal élève.

Plus seulement romancières ou moralistes ou pédagogues, il y a les audacieuses que rien n'arrête. Celles par exemple qui osent écrire l'Histoire comme Mme Thiroux d'Arconville : à cinquante ans, profitant des ressources documentaires de la Bibliothèque du roi où elle a libre accès, elle donne coup sur coup trois ouvrages historiques, Vie du Cardinal d'Ossat, Vie de Marie de Médicis et Histoire de François II. Comme le suggère le changement de titre (on passe de Vie à Histoire), elle s'y montre de plus en plus audacieuse et libre dans son utilisation des sources, d'abord scrupuleusement suivies, puis accompagnées de jugements personnels qui lui valent d'ailleurs des semonces de la critique.

D'autres enfin, plus rares encore, tentées par l'appât du gain ou vraiment inconscientes, osent s'engager dans l'actualité par des écrits séditieux qui peuvent leur valoir à tout moment la prison et la misère. C'est le cas de Mlle Bonafons pour sa collaboration à Tanastes (1747), un petit roman à clé sur les amours de Louis XV et de Mme de Châteauroux : elle paiera son imprudence de quinze ans de réclusion! Ç'aurait pu être le sort d'Anne-Marie de Fauque ${ }^{50}$, si elle n'avait eu la prudence de fuir en Angleterre. Religieuse défroquée, fichée par la police, elle décide, après avoir débuté dans la carrière par des romans dans le goût antique et oriental, de passer à un genre plus lucratif, le pamphlet politique. Elle commence par La dernière guerre des bêtes (1758), une fable animalière inspirée par le ballet fou des alliances dans la guerre de Sept Ans. Elle se lance ensuite dans une Histoire de Mme de Pompadour (1759), pamphlet scandaleux apprécié de Voltaire et qui fit la fortune des colporteurs par son succès. Dans Édition et sédition ${ }^{51}$, Robert Darnton le cite, en effet, dans la liste des ouvrages les plus demandés à l'époque, malgré les tentatives de Mme de Pompadour pour obtenir que les exemplaires soient saisis et brûlés. Mlle Fauque récidivera d'ailleurs avec un autre brûlot à la gloire de Frédéric II, en pleine guerre de Sept Ans. Sous le nom de Mme de Vaucluse, elle finira pourtant ses jours paisiblement et honorablement en Angleterre où elle s'était même trouvé un époux plus

50 Marie-Laure Girou Swiderski, «L'écriture subversive de Mademoiselle de Fauque : La dernière guerre des bêtes et l'Histoire de Mme la marquise de Pompadour», dans M-L. Girou Swiderski (dir). Terrae incognitae de l'écriture féminine, Ottawa/Toronto/ New York, Éditions Legas, 2004, p. 85-95.

51 Robert Darnton, Édition et Sédition, l'Univers de la littérature clandestine au XVIlle siècle, Paris, Gallimard, coll. « NRF / Essais », 1991. 
jeune qu'elle, Lord Stark. Elle fut en relations avec William Beckford, l'auteur de Vatek pour lequel elle effectuait même certains travaux ${ }^{52}$.

\section{Les journalistes}

Reste un type de publication qui donne sans doute plus de visibilité encore et permet de transmettre non seulement un savoir, mais aussi des valeurs : c'est le journal. Le XVIII ${ }^{\mathrm{e}}$ siècle marque les débuts de la presse féminine, des journaux faits non seulement par mais pour les femmes ${ }^{53}$.

Mlle Barbier, déjà fameuse par ses tragédies (qu'on attribuait volontiers à un abbé de ses amis) lance dès 1714 les Saisons littéraires dont la parution s'étendra jusqu'à 1722. Mais c'est surtout Anne Marguerite Dunoyer, huguenote, qui se distingue en ce début de siècle. Mère d'une des premières amantes de Voltaire, elle débute en 1707 avec des Lettres historiques et galantes qu'elle fait paraître en Hollande où elle s'est réfugiée. Le journal emprunte la forme d'une correspondance entre deux amies de longue date, ce qui permet un ton très libre, volontiers frondeur $^{54}$. Il paraît jusqu'en 1717. Entre temps, de 1711 à sa mort, Mme Dunoyer publie un autre périodique qui connaîtra le plus grand succès, la Quintessence des nouvelles historiques, morales, politiques et galantes ${ }^{55}$.

52 Nous remercions vivement Isobel Grundy qui nous a généreusement communiqué l'article suivant, introuvable aujourd'hui : Fatma Moussa-Mahmoud, «Mme Vaucluse, author and femme philosophe of the 18th century». Reprint from Cairo Studies in English, the Bulletin of the Faculty of Arts, Cairo University Press, vol. XXXII, 1978, p. 155-180.

53 Suzan Van Dijk, Traces de femmes. Présence féminine dans le journalisme français $d u$ XVIII siècle, Maarsen, Amsterdam, 1988.

54 Sur Mme Dunoyer et ses Lettres historiques et galantes, voir, entre autres Alain Nabarra, "Correspondances réelles, correspondances fictives: les Lettres historiques et galantes de Mme Dunoyer ou 'la rocambole' d'un 'petit badinage établi d'abord pour le plaisir' " Femmes en toutes lettres, Les épistolières $d u$ XVIII siècle, Oxford, Voltaire Foundation, coll. «Studies on Voltaire and the Eighteenth Century / SVEC », 2000, p. 7-22. Voir aussi Henriette Goldwyn, «Journalisme polémique à la fin du XVII ${ }^{\mathrm{e}}$ siècle : le cas de Mme Dunoyer», dans Colette Nativel (études réunies par), Femmes savantes, Savoirs des femmes, Du crépuscule de la Renaissance à l'aube des Lumières, Genève, Droz, 1999, p. 247-256.

55 Alain Nabarra, «Mme Dunoyer et la Quintessence : la rencontre d'une journaliste et d'un journal", dans Roland Bonnel et Catherine Rubinger (éd.), Femmes savantes et femmes d'esprit, Women Intellectuals of the French Eighteenth Century, Peter Lang, 1994, p. 45-77. 
Ce journal séduit, entre autres, par sa grande variété ; Mme Dunoyer y utilise en effet tous les genres d'écriture : nouvelles et on-dit, jugements séditieux sur l'actualité, récits de voyages et lettres de lectrices, souvent composées par elle-même et où elle se décerne des éloges dithyrambiques, le tout dans un style enlevé et badin. Écrits par des femmes, ces trois périodiques s'adressaient au public en général.

En 1759, par contre, paraît Le journal des dames qui, comme son nom l'indique, vise un lectorat essentiellement féminin. Comptant neuf rédacteurs durant son existence, il est dirigé au début et dans ses dernières années par des hommes. Mais c'est sous l'influence de trois rédactrices remarquables que, de 1761 aux années 1780, le journal prend de l'essor et se distingue des autres périodiques. La première, Mme de Beaumer, huguenote comme Mme Dunoyer, est restée une quasi-inconnue. En Hollande, elle avait déjà écrit des romans et fondé un journal sans lendemain, les Lettres curieuses, instructives et amusantes, inspirées peut-être par l'exemple de Mme Dunoyer. En France, elle était connue des censeurs qui refusaient ses manuscrits trop avancés pour leur goût. En 1761, elle prend la direction du Journal des dames. On peut se demander, comme pour Mme Dunoyer, si son appartenance au protestantisme n'a pas joué un rôle dans sa volonté d'informer ses semblables et de se montrer critique envers les injustices de la société de son temps. Comme le souligne en effet un critique, Mme de Beaumer fera du Journal des dames "un texte sérieux et d'opposition, traitant de problèmes sociaux, prêchant le changement et incitant ses lectrices à abandonner la frivolité, à penser et à cultiver leur esprit ${ }^{56} »$.

Ardente féministe, elle exprime avec force ses convictions et ses dégoûts, surtout à l'égard des hommes, du mariage et de la condition féminine en général. Dans son "Avant-propos » d'octobre 1761, elle annonce : "connaissant tout ce que vaut mon sexe je veux contribuer à sa gloire et à son amusement», et plus loin elle précise sa position revendicatrice: "heureuse de pouvoir venger mon sexe de cette idée injurieuse où sont encore quelques barbares parmi nos concitoyens qui ont de la peine à nous accorder la faculté de penser et d'écrire». On aura noté la virulence de son style. La présentation d'ouvrages récemment parus lui fournit souvent l'occasion de formuler des critiques sur le monde tel qu'il va. Elle dissimule sans doute aussi parfois, par pru-

56 Voir Histoire générale de la presse française, des origines à 1814, PUF, 1969 : «Débuts de la presse féminine», p. 315-318. Soulignons que ces pages sont totalement décalquées sur celles d'Evelyne Sullerot, Histoire de la presse féminine en France, des origines à 1848, Armand Colin, 1966, p. 18-31. 
dence, ses convictions les plus outrées dans des lettres prétendument écrites par des lectrices éclairées, et ses propos évoquent alors bien souvent ceux prêtés aux Précieuses par l'abbé de Pure ${ }^{57}$ dans son roman éponyme.

Son radicalisme ne lui réussira pas : il fait peur aux lectrices et lui vaudra très vite des déboires avec les censeurs. Aussi doit-elle bientôt se défaire du journal en faveur de Mme de Maisonneuve. Cette dernière, bien en cour et à son aise, est beaucoup plus modérée et diplomate, quoique professant elle aussi des convictions féministes. Elle poursuit dans la veine éclectique de Mme de Beaumer. En trois ans, elle quadruple les profits du journal et reçoit une pension du roi. Peu à peu, cependant, elle s'en remet à son assistant, Mathon de la Tour, qui se permet de publier les œuvres les plus subversives du jeune Louis-Sébastien Mercier.

Suspendu pendant les troubles parlementaires, le journal reprend en 1774 avec une troisième directrice, Mme de Prinzen, qui finira de donner au journal une stature imposante. Elle commence par en modifier le titre : c'est désormais Le Journal des dames dédié à la Dauphine par la baronne de Prinzen et de Montanclos. Marquée par la révolution rousseauiste, Mme de Prinzen exhorte les femmes à remplir leurs devoirs de mère, à allaiter leurs enfants et à s'occuper elles-mêmes de leur éducation comme Julie. Sous sa direction, le journal prend un coloris plus nettement pratique, faisant la part belle aux questions d'hygiène (l'inoculation) et d'éducation. Mais, émule des deux précédentes éditrices, la baronne souhaite aussi que les femmes puissent avoir une carrière et s'épanouir intellectuellement. Comme l'affirme son éditorial : «je veux rendre aux femmes la justice que les hommes leur refusent comme à plaisir»; et plus loin encore : «je veux faire savoir que nous pouvons tout savoir car notre esprit est ardent et flexible ${ }^{58} \gg$.

En 1774, elle publie même, expurgé, un conte de Voltaire, Le Crocheteur borgne. Elle cèdera finalement le journal à Mercier qui en accentuera les accents revendicateurs jusqu'à ce que la Révolution mette un terme à sa publication. Il sera alors remplacé par le Journal des dames et des modes, périodique dirigé par un homme, dont le titre marque bien le retour à la frivolité.

Ce qui frappe le plus dans ces exemples, c'est de voir à quel point ces femmes ont su pressentir le potentiel extraordinaire de ce moyen de communication. C'est évident chez Mme Dunoyer pour qui la com- 
plicité avec sa correspondante, moitié réelle, moitié fictive, semble le gage de sa connivence avec les lecteurs. Les rédactrices du Journal des dames, avec des caractères différents, n'en jouent pas moins à fond le jeu de l'échange et de la communication, sans perdre de vue la mission éducative qu'elles se sont donnée. Elles savent d'ailleurs aussi fort bien aller chercher les collaborateurs qui leur permettront de mieux atteindre leurs objectifs.

\section{Femmes et sciences ${ }^{59}$}

À une époque d'intense curiosité intellectuelle, dont l'Encyclopédie reste le modèle insurpassé, en un temps aussi de profonds bouleversements des connaissances scientifiques, sous l'égide entre autres de la révolution newtonienne, les femmes ont montré qu'elles se sentaient bien de leur siècle et n'avaient pas oublié le message cartésien. Elles font leur également la devise kantienne : «Ose savoir». Cet appétit est d'autant plus surprenant que rien, dans leur condition, ni surtout dans leur éducation, ne les dispose à se montrer audacieuses en ce domaine. Au contraire, tous les traités d'éducation sont bardés de mises en garde contre tous les dangers que le goût des sciences ferait courir à la vertu féminine. Pas question pour elles, en effet, de s'intéresser à la médecine ou à l'anatomie, trop contraires à la pudeur. On a vu que même les mathématiques étaient suspectes : que sera-ce alors de la physique ou de la chimie! Passe encore pour les sciences naturelles ( les herbiers ne semblent pas trop dangereux ; en outre, on connaît l'intérêt que Rousseau leur porte) et surtout l'astronomie (contempler les astres ne peut évidemment qu'élever l'âme!). C'est donc en contournant ces multiples interdits que quelques-unes d'entre elles ( et sans doute en reste-t-il bien d'autres encore à découvrir) ont pu satisfaire leur curiosité, et même se tailler une réputation enviable dans ce domaine traditionnellement masculin. Rappelons pourtant qu'aucune n'a pu collaborer à l'Encyclopédie.

À l'époque, les sciences sont regroupées par l'Académie selon trois axes, dont deux surtout progressent remarquablement : outre les Sciences de la terre (géographie, géologie), on distingue les Sciences de la vie (anatomie, médecine, biologie, chimie) et celles de l'Univers (mathé-

59 Je tiens à exprimer ici ma gratitude à M. Patrice Bret, collaborateur au New Dictionary of Scientific Biography, dont l'aide m'a été précieuse pour la rédaction de cette partie; je lui dois, en particulier, la découverte de Mme Picardet dont il est le biographe. 
matiques, physique et astronomie). C'est en rapport avec ces deux derniers axes que nous verrons les femmes se distinguer, la plupart grâce aux traductions, mais d'autres aussi par de véritables avancées scientifiques.

Le premier handicap à surmonter est la totale insuffisance de leur formation. Pour la parfaire, elles doivent compter sur les occasions et les relations. Certains savants, Maupertuis puis Koenig pour Mme Du Châtelet par exemple, les acceptent comme élèves. Dans certaines disciplines, on s'est même ingénié à faciliter leur apprentissage : Fontenelle avait montré le chemin avec ses Entretiens sur la pluralité des mondes (1686); Algarotti lui emboîte le pas quelques décennies plus tard avec Le Newtonianisme des dames (1737) et à la fin du siècle, Lalande publie aussi son Astronomie des dames (1786). Devant l'engouement pour certaines disciplines, l'anatomie par exemple, quelques savants acceptent de donner des cours au Jardin du Roi. C'est là que Mme Thiroux d'Arconville a pu se former en anatomie et même en médecine.

Rares seront celles, comme Mme Du Châtelet, qui chercheront à égaler leurs maîtres et y réussiront presque. Pour beaucoup, dans ce domaine comme dans d'autres, la traduction constitue la voie d'accès et le moyen de se perfectionner, dans certains cas de se faire connaître et même reconnaître par le milieu scientifique.

De toutes façons, ces femmes scientifiques ne sont pas très nombreuses ( sans doute plus qu'on ne croit cependant): dans ce domaine aussi, il existe une forme d'invisibilité. Si elles travaillent avec un savant reconnu (comme Lalande ou Condillac), il n'a pas toujours l'honnêteté de reconnaître l'importance de leur collaboration. Si elles collaborent avec leur époux, c'est à ce dernier qu'on attribue plus volontiers leurs découvertes ou leur contribution. $\mathrm{Si}$, célibataires, elles osent s'aventurer dans ces domaines réputés masculins, sans de grands appuis ou de bonnes relations, leurs chances de se voir reconnues sont presque nulles. Et pourtant, elles ont essayé ${ }^{60}$.

Marie Catherine Biheron (1719-1795), célibataire, illustre bien ces difficultés. Passionnée d'anatomie à une époque où cet engouement se répand, elle eut l'idée de fabriquer des pièces anatomiques en cire pour pallier la difficulté d'étudier sur de vrais squelettes. En 1761, elle publie L'anatomie artificielle, et s'inspirant peut-être de l'invention de Mme du Coudray, célèbre sage-femme qui avait fait breveter le sien

60 Pour situer l'échantillon ci-dessous dans un ensemble plus vaste, voir Jean-Pierre Poirier, Histoire des femmes de science en France, du Moyen Âge à la Révolution, Pygmalion, 2002. 
en 1758, y propose un mannequin articulé pour enseigner l'obstétrique aux futures sages-femmes ${ }^{61}$. On veut l'inviter en Suède et en Russie. Son succès fait des jaloux ${ }^{62}$, aussi s'exile-t-elle un temps en Angleterre où elle enseigne sa technique à la célèbre Mme Tussaud. Elle en revient pour présenter ses inventions à Paris au jeune roi Gustave de Suède. Elle mourra seule et dans la misère après avoir dû, pour conserver sa pension, livrer ses secrets aux révolutionnaires.

Les mathématiques et la physique en tentent plusieurs. La plus célèbre est bien entendu Mme Du Châtelet. Elle a étudié avec Maupertuis, les frères Bernoulli et finalement Koenig. Elle publie ses Institutions de physique et présente anonymement au concours de l'Académie des sciences (comme Voltaire d'ailleurs) un mémoire sur la nature du feu et sa propagation, problème qui va continuer à diviser les savants jusqu'à la Révolution et Lavoisier. Elle ne remporte pas le prix mais son mémoire, Dissertation sur la nature et la propagation $d u$ feu, est jugé digne de la publication, surtout quand on sait qu'il s'agit de l'œuvre d'une femme. Par la suite, elle ose s'opposer à Dortous de Mairan, futur secrétaire de l'Académie des sciences : elle expose les erreurs qu'il a commises en cherchant à réfuter la théorie des forces vives de Leibniz. Cette querelle fait scandale ${ }^{63}$. Voltaire qui n'est pas leibnizien prend le parti de Mairan, tout en se montrant très fier des prouesses scientifiques de la marquise. Elle finira sa carrière par où les autres commencent, en traduisant du latin les Principia de Newton, qu'elle achève en 1749, juste avant de mourir de fièvre puerpérale. Le texte est publié par Voltaire et sert encore aujourd'hui aux rééditions modernes des Éléments de Newton. Celle qui affirmait, dans son Discours sur le bonheur, que seule l'étude pouvait faire accéder les femmes à la gloire, pouvait mourir contente, sûre que cette œuvre lui survivrait. Ce n'est pourtant que très récemment qu'on a commencé à se pencher sur sa carrì̀re scientifique et à s'interroger sur l'importance de sa contribution à l'avancement des sciences. Pendant longtemps, elle ne fut connue que comme la maîtresse tyrannique de Voltaire.

61 Pour subvenir à ses besoins, elle ouvre un cabinet de curiosités où figurent ses inventions et qui sera finalement racheté pour le compte de Catherine de Russie.

62 Plusieurs de ses lettres figurent dans les Franklin Papers, The American Philosophical Society et Yale University, 1954 sq.; voir par exemple en 21-331 une lettre d'octobre 1774.

63 Elisabeth Badinter, "Quand la marquise défie le secrétaire perpétuel», dans Les Passions intellectuelles, Fayard, t. I, 1999, p. 167-186. Voir aussi Badinter, Elisabeth, Emilie, Emilie, l'ambition féminine au XVIII' siècle, Flammarion, 1983. 


\section{Marie-Laure Girou Swiderski}

A la fin du siècle, Sophie Germain ( 1776-1831), célibataire elle aussi, se consacre également aux mathématiques. Elle prend un pseudonyme masculin pour correspondre avec le grand mathématicien Lagrange. Il découvre bientôt sa véritable identité mais, impressionné par ses dons, accepte de la prendre pour élève. Quand il est nommé en Allemagne, elle poursuit seule ses recherches mais, ayant entamé une correspondance avec le mathématicien allemand Gauss, elle change d'orientation pour se consacrer désormais à la question de l'élasticité. Il l'aidera à redresser ses erreurs et en 1815 elle obtiendra enfin le prix de l'Académie. Elle a laissé son nom à une catégorie de nombres premiers et fut la première femme admise à suivre les séances de l'Institut. Ses résultats ont permis de poser les bases de la théorie moderne de l'élasticité. Elle meurt à cinquante-cinq ans d'un cancer du sein.

Traductrice, polygraphe et passionnée de sciences, Mme Thiroux d'Arconville (1720-1805) alterne, comme Mme Du Châtelet, traductions et travaux personnels. Elle s'est dotée de solides compétences en anatomie, en chimie et en médecine. Elle correspond avec le chimiste Macquer qui l'aide dans la correction de ses travaux (on trouve quelques lettres qu'ils échangèrent à la BNF) et est également connue et estimée du minéralogiste, Grimoald Monnet. Elle possède dans sa propriété de Meudon un laboratoire où elle poursuit ses expériences sur les champignons, les mousses et les moisissures. Quand elle traduit les Leçons de chimie de Shaw, elle y joint une ambitieuse Histoire de la chimie qui fut très appréciée de la critique. Forte des multiples expériences qu'elle a menées, elle ose publier un Essai sur la Putréfaction ${ }^{64}$, sujet capital en un temps qui ignore la réfrigération. Elle traduit le Traité d'ostéologie de Monro, dont elle corrige les erreurs et refait les croquis ( son dessin du squelette féminin restera utilisé jusqu'à la Révolution et on le trouvait encore dans les années 1950 dans certains manuels de médecine).

L'astronomie a partie liée avec les mathématiques et l'horlogerie. $C^{\prime}$ est l'autre discipline scientifique très à la mode. Le XVII ${ }^{\mathrm{e}}$ siècle déjà avait vu des femmes s'y illustrer. Reine Lepaute (1723-1788) s'y distinguera tout particulièrement $t^{65}$. Collaborant avec son mari horloger, elle entre en relations avec Lalande avec qui elle écrit d'abord un traité d'horlogerie. Il l'engage pour collaborer avec Clairaut au calcul

64 On trouve une bonne étude de l'intérêt de ce texte et de la rigueur méthodique de Mme Thiroux d'Arconville dans le livre de Sabine Barles, La Ville délétère, médecins et ingénieurs dans l'espace urbain, XVIII'-XIX' siècle, Champ Vallon, 1999, p. 65-67.

65 Elisabeth Badinter en parle, ainsi que de Mme Thiroux d'Arconville d'ailleurs, dans Les Passions intellectuelles, t. II, «Femmes savantes», Fayard, 2002, p. 250-262. 
du retour de la comète de Halley. Ils prédisent son retour pour avril 1759 , la comète arrive en fait mi-mars, soit une erreur de deux semaines dont on leur tient rigueur dans les milieux scientifiques. A partir de là, elle se consacre au calcul astronomique et participe à la rédaction des éphémérides destinés à la navigation. A l'inverse de Clairaut, Lalande lui rend publiquement hommage. Ses travaux sont d'ailleurs publiés par l'académie des sciences et elle devient membre de l'Académie de Béziers. En reconnaissance de son apport à l'astronomie, un des cratères de la lune porte son nom.

Mariée au chimiste Lavoisier, Marie-Anne Paulze (1758-1836) se fait sa collaboratrice ${ }^{66}$. En aidant son mari et en fréquentant d'autres savants de l'époque, elle réussit à se doter d'une solide formation de chimiste $^{67}$. Prenant modèle sur une grande traductrice scientifique de l'époque, Mme Picardet, dont nous allons parler plus bas, elle traduit avec son aide le Traité de Phlogistique de Kirwan, chimiste irlandais. Tout au long de la carrière de son mari jusqu'à son exécution en 1794, elle le seconde dans ses travaux. Passionnée de peinture et de dessin ${ }^{68}$ (elle est l'élève de David), elle illustre les ouvrages de son mari. Ainsi le Traité élémentaire de chimie, paru en 1790, est orné de bois gravés de sa main. Elle collabore aussi au journal Les annales de chimie où elle rend compte des ouvrages étrangers. En 1792, elle publie un autre article de Kirwan sur les acides que son mari annotera. Après la mort de Lavoisier, elle se consacre à la publication de ses travaux, dont les Mémoires de chimie, restés inachevés.

Claudine Picardet, dijonnaise (1735-1820), est la plus remarquable par l'importance de ses réalisations comme traductrice. Elle avait suivi les cours de Guyton de Morveau, le collaborateur de Buffon. Polyglotte, elle traduira des ouvrages de chimie, de minéralogie et même d'astronomie à partir de cinq langues modernes et du latin. Elle publie ses traductions d'abord anonymement mais à partir de 1786, elle signe et Lalande inscrit son nom dans le Journal des savants. Elle publie aussi ses traductions dans les Observations de physique. Une de ses plus belles

66 Jean-Pierre Poirier, La Science et l'amour, Madame Lavoisier, Flammarion, 2004.

67 Dans une note de ses Travels in France during the years 1787,1788,1789, Arthur Young loue la compétence scientifique de Mme Lavoisier : cité dans l'Introduction de Roland Bonnel et Catherine Rubinger (éd.), Femmes savantes et femmes d'esprit. Women Intellectuals of the French Eighteenth Century, New York, Peter Lang, coll.«Eighteenth Century French Intellectual History», 1994, p. 29.

68 Madeleine Pinault-Sorensen, «Mme Lavoisier, dessinatrice et peintre», La revue $d u$ Musée des Arts et Métiers, mars 1994, p. 23-25. 
réalisations est la traduction des Mémoires de chimie de l'allemand Schelle qu'elle annote avec l'aide de Guyton de Morveau. A partir de 1785, elle travaille en météorologie avec Mme Lavoisier et l'aide dans sa traduction de Kirwan. Veuve en 1796, elle se remarie avec son ancien professeur et devient sous l'Empire la baronne Guyton-Morveau. Elle a contribué au développement de la chimie minérale, a collaboré à la création de nombreuses revues scientifiques et est une des premières à se voir décerner le statut d'auteur pour ses traductions.

J'aimerais terminer en présentant une dernière femme qui s'est illustrée dans un domaine scientifique, d'autant que le récit de sa vie semble le canevas d'une comédie du XVIII ${ }^{\mathrm{e}}$ siècle. Jeanne Baret ${ }^{69}$ (1740-1807) d'origine modeste, est formée aux sciences naturelles par son amant, le naturaliste Philibert Commerson. Il est désigné pour accompagner Bougainville dans son périple autour du monde où il est chargé d'observer et de classifier la flore des pays visités. Il est aidé dans sa collecte et ses observations par son valet, Baret, qui recueille les spécimens et exécute force croquis. Il s'agit de Jeanne dont le subterfuge est éventé à Tahiti par un indigène libidineux. Diderot relate d'ailleurs cet épisode haut en couleurs dans le Supplément au voyage de Bougainville ${ }^{70}$. Arrivés à l'île Maurice, Commerson meurt et Jeanne, se retrouvant seule, ouvre un cabaret. Elle se fait expulser de l'île pour avoir servi de l'alcool le dimanche. Après s'être mariée avec un soldat, elle rentre en France. Ici les versions diffèrent : certains prétendent que tout ou presque fut perdu. D'autres assurent qu'elle rapporta avec elle 34 caisses contenant des spécimens de 5000 plantes dont 300 inconnues (la fameuse bougainvillée, découverte au Brésil, en faisait partie et a inspiré un roman ${ }^{71}$ ). Elle fit don de ce butin au Jardin du Roi, futur Jardin des Plantes et Musée national d'histoire naturelle.

Voici donc un domaine où, de toute évidence, les femmes ont joué un rôle important dans l'avancement et la transmission des connaissances. Comme pour les salons, dans le cadre de ces activités scientifiques, les femmes se trouvent avoir occupé le plus souvent une place d'accompagnatrice, parfois de maïeuticienne; on peut penser à la mystérieuse Mlle Ferrand, collaboratrice géniale de Condillac, l'inventrice

69 Outre de nombreuses références sur Internet, et le récit de Bougainville dans son Voyage autour du monde, on peut lire sa biographie: Henriette Dussourd, La Première Femme autour du monde, Pottier, Moulins, 1987.

70 Diderot, "Supplément au voyage de Bougainville ou dialogue entre A. et B.», CEuvres philosophiques, Garnier, 1964. L'épisode est narré p. 474.

71 Voir Fanny Deschamps, La Bougainvillée, t.1, L'allée du roi, Albin Michel, 1986. 
de la statue animée qui permit à ce dernier de poser les bases du sensualisme et que Larry Bongie a si magistralement redécouverte ${ }^{72}$.

Alors : la République des Lettres au féminin? Pas tout à fait encore. Il a manqué à toutes ces femmes les réseaux, les relations, les institutions qu'elles ont si efficacement contribué à procurer aux hommes. C'est seulement à la fin du siècle qu'on peut déceler entre elles quelques traces d'intertextualité. Ce qui distingue les hôtesses et qui explique peut-être qu'on s'acharne à minimiser leur importance, $c^{\prime}$ est bien, sans vouloir en faire des exemples, ce pouvoir parallèle, cette « chaîne secrète » qu'elles font courir d'un bout du siècle à l'autre, l'art d'inspirer la société en animant un salon.

Nous les avons vues présentes sur tous les fronts de l'activité littéraire et intellectuelle de leur temps. Certes, nous avons perdu à tout jamais la possibilité de prendre la mesure exacte de leur influence en les écoutant parler ou en les voyant animer leur salon. Mais nous avons leurs lettres, équivalent écrit de la conversation, pour retrouver des traces de leur esprit, de leur intelligence, de leur sens aigu des réalités sociales.

Du fait du tabou de la publication, à cause aussi des délais qu'elle implique, la plupart de leurs œuvres, à part les romans, sont parues posthumes. Si bien que le tableau de l'activité littéraire féminine au XVIII siècle que nous pouvons tracer aujourd'hui n'a presque plus rien de commun avec l'image que pouvaient s'en faire les contemporains. Et encore, nous n'avons parlé ici ni des poètes ni des dramaturges! Ce que nous avons perdu à l'oral, nous l'avons regagné amplement par l'écrit. Très peu de gens au XVIII siècle ont pu connaître, par exemple, la correspondance de Mme de Graffigny. Il semble que Sainte-Beuve lui-même ignore celle de Mme de Charrière avec Benjamin Constant. Et qui aurait pu deviner alors dans Julie de Lespinasse, la parfaite hôtesse, l'amante tourmentée de Mora et Guibert ? Toutes ces formes d'invisibilité n'existent plus pour nous qui avons la chance aujourd'hui de connaître de plus en plus, de mieux en mieux, ces femmes qui ont dû affronter tant d'obstacles simplement pour pouvoir exercer la force de leur esprit et de leurs talents. Le temps n'est-il pas venu alors de les intégrer enfin dans le canon et de les traiter comme parties prenantes de l'épopée des Lumières? Qu'elles l'aient appuyé ou combattu (comme Mme de La Ferté-Imbault), elles apparaissent bien, en effet, comme des membres à part entière de ce mouvement par leur soif de savoir et plus

72 Laurence L. Bongie, Diderot's 'femme savante', Oxford, Voltaire Foundation, coll. «Studies on Voltaire and the Eighteenth century», 1977. 
28 Marie-Laure Girou Swiderski

encore par leur volonté inlassable de communiquer ce savoir par tous les moyens à leur disposition, la conversation et tous ses équivalents écrits.

MARIE-LAURE GIROU SWIDERSKI

Université d'Ottawa 